\title{
Temperature Cross-Sensitivity for Highly Refractive Index Sensitive Nanocoated Long-Period Gratings
}

\author{
J. Grochowski ${ }^{a}$, M. Myśliwiec ${ }^{a}$, P. Mikulic ${ }^{b}$, W.J. Bock ${ }^{b}$ And M. Śmietana ${ }^{a, *}$ \\ ${ }^{a}$ Institute of Microelectronics and Optoelectronics, Warsaw University of Technology \\ Koszykowa 75, 00-662 Warsaw, Poland \\ ${ }^{b}$ Centre de Recherche en Photonique, Université du Québec en Outaouais \\ 101 Rue St Jean Bosco, Pavillon Lucien Brault, Gatineau, Québec, J8X 3X7, Canada
}

\begin{abstract}
There is a demand for highly refractive index sensitive devices simultaneously insensitive to temperature $(T)$ changes. We investigate both $T$ and refractive index sensitivity of long-period gratings coated with $100 \mathrm{~nm}$ thin high refractive index $(n \approx 1.9$ at $\lambda=1550 \mathrm{~nm})$ silicon nitride $\left(\mathrm{SiN}_{x}\right)$ film. The long-period gratings with period of $500 \mu \mathrm{m}$ were induced in standard Corning SMF28 fibre using electric arc and nanocoated with SiN ${ }_{x}$ by radio frequency plasma enhanced chemical vapour deposition method. As a sensorial effect we investigated resonance shift with temperature variations (from 30 to $70^{\circ} \mathrm{C}$ ) for measurement in deionized water $\left(n_{\mathrm{D}}=1.33\right.$ ) and glycerine $\left(n_{\mathrm{D}}=1.46\right)$. In case of measurements in water for resonance observed at $\lambda=1510 \mathrm{~nm}$, refractive index sensitivity of $-85 \mathrm{~nm} / \mathrm{RIU}$ and temperature sensitivity of $0.09 \mathrm{~nm} /{ }^{\circ} \mathrm{C}\left(\mathrm{H}_{2} \mathrm{O}\right)$ were obtained which gives refractive index- $T$ sensitivity ratio $(\mathrm{RI} / T)$ of $-944^{\circ} \mathrm{C} / \mathrm{RIU}$. Refractive index and $T$ sensitivity of sensor covered with $\mathrm{SiN}_{x}$ film increased to $-618 \mathrm{~nm} / \mathrm{RIU}$ and $0.22 \mathrm{~nm} /{ }^{\circ} \mathrm{C}$, respectively. Thanks to nanocoating deposition, refractive index- $T$ sensitivity ratio was improved by factor of 3 . In case of measurements in glycerine significant influence of glycerine's thermo-optic coefficient on obtained results was observed. It is worth noticing that $\operatorname{SiN}_{x}$ coating stabilizes sensing behaviour of the sensor.
\end{abstract}

DOI: $10.12693 /$ APhysPolA.124.421

PACS: 42.70.Ce, 42.79.Gn, 07.07.Df, 81.20.Fw

\section{Introduction}

Biological and chemical sensors are attracting much attention because of their application in medical diagnostics [1], development of novel drugs and testing food for hazardous substances [2]. Currently the majority of commercially available biologic species (e.g. DNA, antigens) sensing schemes are based on fluoroimmunoassay, i.e. fluorescent labelling of target measurand. This scheme requires additional staff involvement along with extended analysis time and higher costs. For disadvantages and the need of real-time sensing, label-free bio-detection is being extensively researched. In this field optical fibre sensors are showing advantages thanks to their ability to provide fast, reliable results accompanied by relatively low production costs [3]. The most important aspect of bio-sensing is fabrication of devices characterized by maximum available refractive index (RI) sensitivity [4].

Long-period grating (LPG) is an optical device presented for a first time in 1995 by Vensarkar et al. [5]. LPG is a periodic modulation (period typically of 140 $770 \mu \mathrm{m}$ ) of refractive index of optical fibre's core. Coupling of guided core mode to the forward-propagating $m$ cladding modes, where $m$ represents order of the cladding modes, results in a transmission spectrum consisting of attenuation peaks appearing at wavelengths $\lambda_{m}$ satisfying equation

$$
\lambda_{m}=\left(n_{\mathrm{eff}}^{\text {core }}-n_{\mathrm{eff}, m}^{\text {clad }}\right) \Lambda,
$$

${ }^{*}$ corresponding author; e-mail: msmietan@elka.pw.edu.pl where $\Lambda$ is the grating period, $n_{\mathrm{eff}}^{\text {core }}$ and $n_{\mathrm{eff}, m}^{\mathrm{clad}}$ are effective refractive indices of core and cladding modes, respectively [6]. External medium RI causes changes of effective refractive indices of the cladding modes leading to RI-sensitivity of LPGs. Unfortunately, because of the difference in thermo-optic coefficients of the core and the cladding materials, LPGs are also sensitive to temperature variations. This property used for the fabrication of temperature sensors is undesirable in biological and chemical label-free sensor design. Many approaches to overcoming the temperature cross-sensitivity of LPGs were proposed. Most of them employ complex design of the sensing element, where next to LPG other structures showing different temperature sensitivity are used. Such approach has been demonstrated by simultaneous measurement of temperature and RI using LPG and fibre Bragg grating (FBG) [7], structure composed of bare LPG and Ag-coated LPG [8], sandwiched LPG sensor design [9] and Michelson refractometer with compound cavity based on LPG [10]. There have been also reported attempts of using special fibres [11] or proper selection of cladding modes [12] for reduction of temperature sensitivity. In our previous work we have shown that the RI-sensitivity can be greatly improved by application of high-refractive-index silicon nitride $\left(\mathrm{SiN}_{x}\right)$ thin overlay [13]. Because of the fact that external medium RI affects only $n_{\mathrm{eff}, m}^{\text {clad }}$, deposition of the nanocoating significantly modifies RI-sensitivity of LPG. Such procedure allows for LPG-based temperature measurements which can be performed in liquids showing different RI [13].

In this work we study possibility of increasing the RI-sensitivity and thus modification of the tempera- 
ture cross-sensitivity. The investigated sensing structure is based on LPG with silicon nitride $\left(\mathrm{SiN}_{x}\right)$ thin film nanocoating deposited on device side walls. Influence of the coating on RI to temperature sensitivity ratio is discussed.

\section{Experimental details}

To fabricate our optical refractive index sensors, standard SMF28 single mode $\left(d_{\text {core }}=8.2 \mu \mathrm{m}, d_{\text {cladding }}=\right.$ $125 \mu \mathrm{m})$ fibres from Corning were used. Outer polymer coating was removed mechanically from the fibres at the area, where LPGs were about to be written. Prior to fabrication of LPGs, fibres were cleaned in organic solvents. LPGs were fabricated using electric arc discharge method [14]. Gratings parameters were: length $L=50 \mathrm{~mm}$, period $\Lambda=500 \mu \mathrm{m}$. After RI and temperature sensitivity measurements, on some of the sensors $100 \mathrm{~nm}$ thin $\mathrm{SiN}_{x}$ films were deposited using radio frequency plasma enhanced chemical vapor deposition (RF PECVD) technique [15]. Oxford Instruments Plasmalab 80Plus was used. In order to obtain uniformity of $\mathrm{SiN}_{x}$ film around the circumference of optical fibre, custom made fibre-holder was used. It allowed to maintain constant distance between the fibre itself and electrode during deposition process. These films were simultaneously deposited on reference silicon substrates in order to perform refractive index measurement by means of spectroscopic ellipsometry using Horiba Jobin Yvon UVISEL ellipsometer working in wavelength range from 260 to $830 \mathrm{~nm}$.

The sensitivity of fabricated sensors is hereby defined as a shift of LPG resonant wavelength caused by increase either of external medium's RI or ambient temperature. In order to evaluate the performance of the sensor we introduce $S_{\mathrm{RI}}$ and $S_{T}$ coefficients to describe sensitivity mentioned above and defined as follows:

$$
S_{\mathrm{RI}}=\frac{\Delta \lambda}{\Delta n}=\frac{[\mathrm{nm}]}{[\mathrm{RIU}]}, \quad S_{T}=\frac{\Delta \lambda}{\Delta T}=\frac{[\mathrm{nm}]}{\left[{ }^{\circ} \mathrm{C}\right]},
$$

where $\Delta \lambda$ is peak shift, $\Delta T$ is temperature change and $\Delta n$ is RI change.

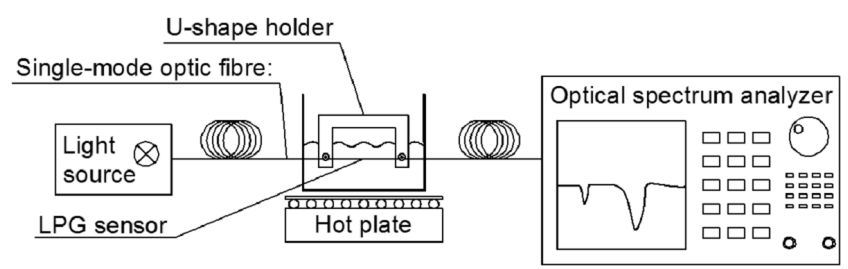

Fig. 1. Experimental setup used to measure RI and temperature sensitivity of fabricated sensor.

Figure 1 shows experimental setup used to measure $S_{\mathrm{RI}}$ and $S_{T}$ of fabricated sensor. The sample U-shape holder allowed for maintenance of constant tension of the grating during the measurements. White light used in the experiment is Yokogawa AQ4305 and optical spectrum analyzer is Yokogawa AQ6370B. Several solutions of water and glycerine were prepared and the range of RI values from about 1.33 up to 1.40 were obtained. Measurements of temperature sensitivity were executed in deionized water $\left(n_{\mathrm{D}}=1.3330\right)$ and in glycerine $\left(n_{\mathrm{D}}=1.46\right)$ in the temperature range from about 30 up to $70^{\circ} \mathrm{C}$. Refractive index of liquids in which $S_{\mathrm{RI}}$ was evaluated was measured using Reichert A200 refractometer and temperature for $S_{T}$ measurements was controlled by standard laboratory hotplate and its value was confirmed by $\mathrm{Ni}-\mathrm{Cr}$ (type $\mathrm{K}$ ) thermocouple placed next to the LPG.

\section{Results and discussion}

Firstly, RI sensitivity of bare and nanocoated LPG-based sensors were measured. It is known that highest sensitivity of LPG can be obtained for resonant peaks closest to specific dispersion turning point (DTP) of corresponding modes coupling. As it was previously reported, for grating period of about $500 \mu \mathrm{m}$, DTP of $\mathrm{LP}_{06}$ occurs at $\lambda \approx 2 \mu \mathrm{m}[16]$. Therefore only the shift of peak with the highest resonance wavelength in the available spectrum of the sensor was analysed, which offers the highest sensitivity at investigated conditions.

Figure 2 shows transmission spectra obtained from measurements. It is clear that for bare sensor sensitivity is low, but the shape of the peak is constant and shifting towards shorter wavelengths with the increase of external RI (Fig. 2a). The situation is slightly different with spectra set for the device nanocoated with $\mathrm{SiN}_{x}$ (Fig. $2 \mathrm{~b})$. High value of $n$ of the nanocoating $(n \approx 1.9$ at $\lambda=1.55 \mu \mathrm{m})$ results in an initial shift of observed resonance wavelength from $1538 \mathrm{~nm}$ to $1611 \mathrm{~nm}$. What is more, with increase of external RI not only does the peak shift towards shorter values of $\lambda$, but its amplitude is increasing as well from $-2 \mathrm{~dB}$ for $n_{\text {external }}=1.33$ up to $-5 \mathrm{~dB}$ for $n_{\text {external }}=1.39$. The phenomenon is related to transition of the modes discussed in detail elsewhere [17].

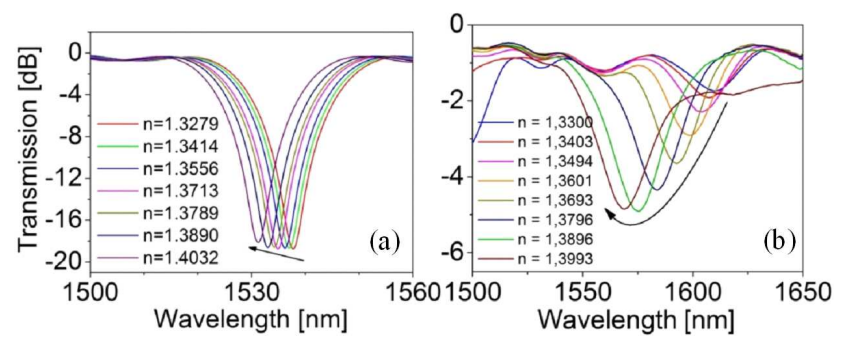

Fig. 2. Transmission spectrum in different RI of (a) bare and (b) $\mathrm{SiN}_{x}$ nanocoated LPGs.

Figure 3 presents plots of measured resonance wavelength shift versus RI values. Linear fit applied to these plots allowed to obtain $S_{\mathrm{RI}}$ values for bare (Fig. 3a) and coated (Fig. 3b) devices and the values of RI-sensitivity are $-85 \mathrm{~nm} / \mathrm{RIU}$ and $-618 \mathrm{~nm} / \mathrm{RIU}$, respectively. Therefore application of $\mathrm{SiN}_{x}$ nanocoating results in increase of RI sensitivity by a factor of 7.2 . 


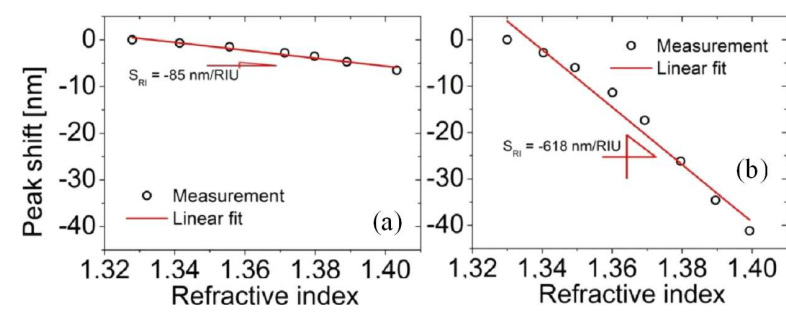

Fig. 3. RI sensitivity plots for (a) bare and (b) $\operatorname{SiN}_{x}$ nanocoated LPGs.

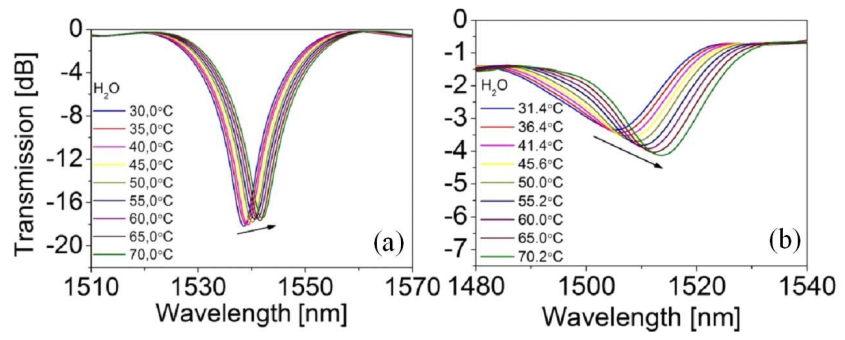

Fig. 4. Transmission spectrum in different $\mathrm{H}_{2} \mathrm{O}$ temperatures for (a) bare and (b) $\operatorname{SiN}_{x}$ nanocoated LPGs.

Next, temperature sensitivity of both sensors was measured. Transmission spectra from these experiments are presented in Fig. 4. In both cases resonance wavelength shift is uniform throughout the whole temperature range and its amplitude is about 6 times smaller for nanocoated sensor. For bare sensor peak shift is relatively small and increases after application of thin $\mathrm{SiN}_{x}$ film. Figure 5 shows $S_{T}$ values corresponding to spectra from Fig. 4 . It can be seen that points denoting experimental values confirm good linear relation between resonance wavelength and $\mathrm{H}_{2} \mathrm{O}$ temperature. Linear fitting results in $S_{T}$ values of $0.09 \mathrm{~nm} /{ }^{\circ} \mathrm{C}$ and $0.22 \mathrm{~nm} /{ }^{\circ} \mathrm{C}$ for bare and nanocoated $\mathrm{LPG}$, respectively. This indicates that temperature sensitivity of the LPG increased more than twice due to deposition of $\mathrm{SiN}_{x}$ film on LPG side walls. The increase of the temperature sensitivity is related to thermo-optic coefficient of water, which is negative. Due to high RI sensitivity of $\mathrm{SiN}_{x}$ nanocoated LPG, decrease of the RI of water with temperature induces additional red shift of the resonances, when compared to bare LPG [13].

$S_{T}$ observations were performed not only in water, but also in glycerine $\left(n_{\mathrm{D}}=1.46\right)$. Transmission measurements when the sample is immersed in glycerine are

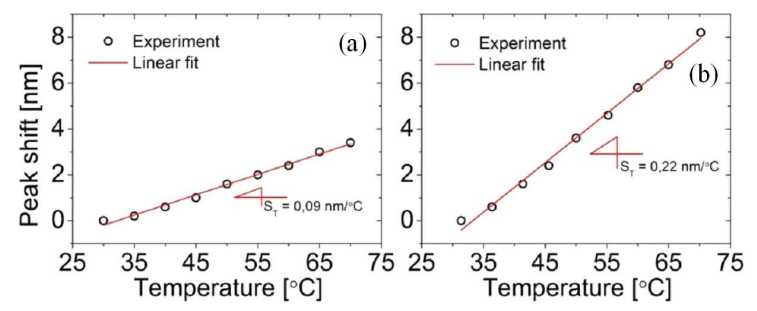

Fig. 5. Temperature sensitivity measured in $\mathrm{H}_{2} \mathrm{O}$ for (a) bare and (b) $\mathrm{SiN}_{x}$ nanocoated LPGs. shown in Fig. 6. Bare LPG sensor reaction to temperature change (Fig. 6a) was rapid and the envelope of the spectrum is significantly distorted when temperature of glycerine is changing from 45 to $55^{\circ} \mathrm{C}$. On the other hand, when the LPG is nanocoated with $\mathrm{SiN}_{x}$ overlay, its response to temperature changes in glycerine is lowered (Fig. 6b). Plot of peak shift versus temperature for glycerine measurement of uncoated sensor shows three different temperature ranges with different values of $S_{T}$ obtained from the slope of linear interpolation of measurement data (Fig. 7a). There is low sensitivity from 30 to $45^{\circ} \mathrm{C}$ and from 55 to $70^{\circ} \mathrm{C}$. Corresponding values of $S_{T}$ are $0.38 \mathrm{~nm} /{ }^{\circ} \mathrm{C}$ and $1.31 \mathrm{~nm} /{ }^{\circ} \mathrm{C}$, respectively. The rapid peak shift occurring when glycerine is heated from 45 to $55{ }^{\circ} \mathrm{C}$ results in $S_{T}=10.73 \mathrm{~nm} /{ }^{\circ} \mathrm{C}$. The fact of presence of these three temperature ranges, each characterized by almost linear fit of measurement data, suggests that our bare LPG sensor detects not only the temperature changes of glycerine medium, but in fact the actual thermo-optic effect known to be strong for this liquid.

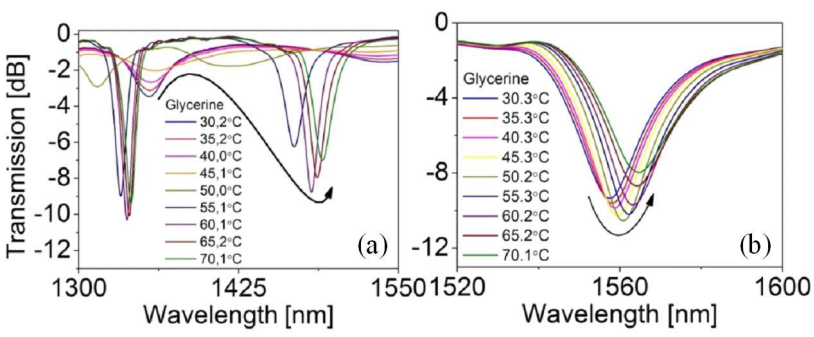

Fig. 6. Transmission spectrum in different $T_{\mathrm{GLYC}}$ of (a) bare and (b) nanocoated LPG sensor.

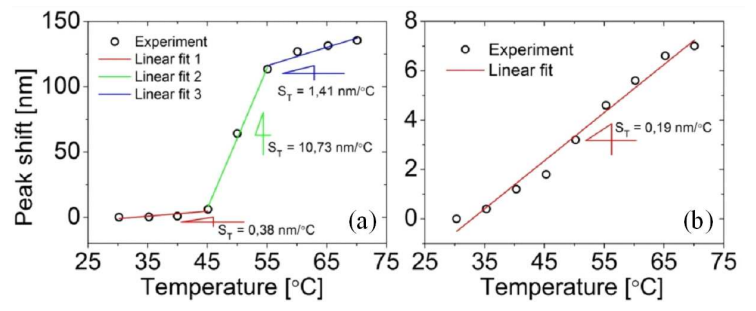

Fig. 7. Temperature sensitivity plots in glycerine of fabricated device for (a) bare and (b) coated with $\mathrm{SiN}_{x}$ LPG sensor.

Figure $7 \mathrm{~b}$ shows that after nanocoating LPG sensor with thin $\mathrm{SiN}_{x}$ films, sensing behaviour is significantly stabilized and values of $S_{T}$ are equalized in the whole temperature range. What is more, temperature sensitivity (i.e. $S_{T}$ value) is lower than any one obtained for uncoated LPG. For RI sensitivity as well as temperature sensitivity measurements, every time, deposition of thin silicon nitride film affects obtained results in different manner. Therefore it is difficult to evaluate results by means of RI-temperature cross-sensitivity of fabricated sensor. For that reason we assess quality of operation of our sensors using $S_{\mathrm{RI}} / S_{T}$ ratio. It is simple to 
estimate whether device's parameters are improved by evaluating this simple number. For example even if simultaneous increase of both $S_{\mathrm{RI}}$ and $S_{T}$ are occurring, when one of these changes is more significant than the other, if the $S_{\mathrm{RI}} / S_{T}$ ratio is increased, then the overall cross-sensitivity is improved. All sensitivity measurements outcomes together with calculated cross-sensitivity ratios are given in Table.

TABLE

$S_{\mathrm{RI}}$ values of both bare and coated with $\mathrm{SiN}_{x}$ sensors as well as $S_{T}$ values obtained from measurements in both $\mathrm{H}_{2} \mathrm{O}$ and glycerine. Resulting $S_{\mathrm{RI}} / S_{T}$ ratios are also shown.

\begin{tabular}{c|c|c|c}
\hline \hline & $\begin{array}{c}\text { Tempera- } \\
\text { ture range } \\
{\left[{ }^{\circ} \mathrm{C}\right]}\end{array}$ & Bare & $\begin{array}{c}\mathrm{SiN}_{x} \\
\text { nano- } \\
\text { coated }\end{array}$ \\
\hline$S_{\mathrm{RI}}[\mathrm{nm} / \mathrm{RIU}]\left(n_{\mathrm{D}}=1.33-1.40\right)$ & & -85 & -618 \\
\hline$S_{T} \mathrm{H}_{2} \mathrm{O}\left[\mathrm{nm} /{ }^{\circ} \mathrm{C}\right]$ & $30-70$ & 0.09 & 0.22 \\
\hline$S_{T}$ glycerine $\left[\mathrm{nm} /{ }^{\circ} \mathrm{C}\right]$ & $30-45$ & 0.38 & \\
& $45-55$ & 10.73 & 0.19 \\
\hline$S_{\mathrm{RI}} / S_{T}$ ratio $\mathrm{H}_{2} \mathrm{O}\left[{ }^{\circ} \mathrm{C} / \mathrm{RIU}\right]$ & $55-70$ & 1.41 & \\
\hline$S_{\mathrm{RI}} / S_{T}$ ratio glycerine $\left[{ }^{\circ} \mathrm{C} / \mathrm{RIU}\right]$ & $30-70$ & -944.44 & -2809.09 \\
\hline & $50-45$ & -223.68 & \\
& $55-70$ & -60.28 &
\end{tabular}

It clearly indicates the fact that $\mathrm{SiN}_{x}$ nanocoating improves operation of the LPG based-sensors. RI sensitivity $\left(S_{\mathrm{RI}}\right)$ is improved approximately 7 times as a result of thin $\mathrm{SiN}_{x}$ overlay deposition. Simultaneously temperature sensitivity is increased by a factor of 2 for measurements performed in deionized water.

This means that $S_{\mathrm{RI}} / S_{T}$ ratio is nearly tripled as a result of film deposition and therefore the sensor performance is tripled as well. We do not evaluate results for $S_{T}$ measurements in glycerine because of nonlinear behaviour of peak shift with increasing temperature. However, it can be pointed out that $\mathrm{SiN}_{x}$ nanocoating also improved this effect, and for nanocoated LPG sensor, $S_{T}$ measurement data for glycerine could be successfully linearly fitted which resulted in $S_{T}=0.19 \mathrm{~nm} /{ }^{\circ} \mathrm{C}$.

\section{Conclusion}

In the paper we discuss cross-sensitivity effect for LPG-based refractive index sensors fabricated by means of arc-discharge method. The sensors were successfully coated with $\mathrm{SiN}_{x}$ thin overlay by RF PECVD technique in order to improve their refractive index-temperature cross-sensitivity. Temperature and RI sensitivity were measured by means of peak shift before and after thin silicon nitride film deposition. Application of $\mathrm{SiN}_{x}$ increased RI as well as temperature sensitivity in water by a factor of 7.2 and 2, respectively. Temperature sensitivity measurements performed in glycerine $\left(n_{\mathrm{D}}=1.46\right)$ revealed significant influence of this medium's thermo-optic coefficient on obtained results. In that case deposition of $\mathrm{SiN}_{x}$ film also greatly improved sensing behaviour of the sensor and lowered its response to temperature changes of glycerine. In order to evaluate overall performance of the sensors, temperature/RI ratio was calculated for bare and coated LPG sensors. It is shown that $\mathrm{SiN}_{x}$ nanocoating successfully decreased cross-sensitivity by factor of 3 in RI range from $n_{\mathrm{D}}=1.33$ to 1.40 .

\section{Acknowledgments}

The authors gratefully acknowledge support for this work from the National Centre for Research and Development of Poland within the LIDER program, and the European Union in the framework of the Innovative Economy Operational Program through the Foundation for Polish Science Homing Plus Program.

\section{References}

[1] T. Claes, J. Girones Molera, K. De Vos, E. Schacht, R. Baets, P. Binstman, IEEE Photon. J. 1, 197 (2009).

[2] J. Hyun Soo, P. Kwan No, P.K. Jun, S. Sang Jun, J.K. Oh, H. Young-Geun, L. Kyung Shik, Opt. Expr. 17, 3855 (2009).

[3] M. Espinosa Bosch, A.J. Ruiz Sanchez, F. Sanchez Rojas, C. Bosch Ojeda, Sensors 7, 797 (2007).

[4] Y. Zhang, S. Gao, A. Ping Zhang, IEEE Photon. J. 4, 2340 (2012).

[5] A.M. Vengsarkar, P. Lemaire, B. Judkins, V. Bhatia, T. Erdogan, J. Sipe, J. Lightwave Technol. 14, 58 (1996).

[6] X. Chen, K. Zhou, L. Zhang, I. Bennion, Appl. Opt. 46, 451 (2007).

[7] X. Shu, B.A.L. Gwandu, Y. Liu, L. Zhang, I. Bennion, Opt. Lett. 26, 774 (2001).

[8] T. Allsop, R. Neal, D. Giannone, D.J. Webb, D.J. Mapps, I. Bennion, Appl. Opt. 42, 3766 (2003).

[9] A.P. Zhang, L.-Y. Shao, J.-F. Ding, S. He, IEEE Photon. Technol. Lett. 17, 2397 (2005).

[10] A. Van Brakel, P.L. Swart, Opt. Eng. 44, 020504 (2005).

[11] H. Dobb, K. Kalli, D.J. Webb, Electron. Lett. 40, 657 (2004).

[12] T. Allsop, D.J. Webb, I. Bennion, J. Lightwave Technol. 21, 264 (2003).

[13] M. Śmietana, W.J. Bock, P. Mikulic, Measur. Sci. Technol. 22, 115203 (2011).

[14] M. Smietana, W.J. Bock, P. Mikulic, J. Chen, Measur. Sci. Technol. 22, 015201 (2011).

[15] M. Smietana, W.J. Bock, P. Mikulic, J. Chen, Sensors 10, 11301 (2010).

[16] X. Shu, L. Zhang, I. Bennion, J. Lightwave Technol. 20, 255 (2002).

[17] P. Pilla, V. Malachovská, A. Borriello, A. Buosciolo, M. Giordano, L. Ambrosio, A. Cutolo, A. Cusano, Opt. Expr. 19, 512 (2011). 